\title{
Alpha fractional frequency Laplace transform through multiseries
}

\author{
Meganathan Murugesan', Thabet Abdeljawad ${ }^{2,3,4^{*}} \mathbb{D}$, Britto Antony Xavier Gnanaprakasam and
} Fahd Jarad ${ }^{5}$

\author{
"Correspondence: \\ tabdeljawad@psu.edu.sa \\ ${ }^{2}$ Department of Mathematics and \\ General Sciences, Prince Sultan \\ University, Riyadh, Saudi Arabia \\ ${ }^{3}$ Department of Medical Research, \\ China Medical University, Taichung, \\ Taiwan \\ Full list of author information is \\ available at the end of the article
}

\begin{abstract}
Our main goal in this work is to derive the frequency Laplace transforms of the products of two and three functions with tuning factors. We propose the Laplace transform for certain types of multiseries of circular functions as well. For use in numerical results, we derive a finite summation formula and $m$-series formulas. Moreover, we discuss various explanatory examples.
\end{abstract}

MSC: 39A70; 47B39; 39A10; 44A10

Keywords: Circular function; Multiseries; Laplace transform; Fractional frequency; Tuning factor

\section{Introduction}

Digital signal processing (DSP) has revolutionized many areas in science and engineering such as space, medicine, commerce, military, technology, and communication. The Laplace transform (LT) and discrete Laplace transform (DLT) effectively change a signal (function) from time domain to frequency domain with the factor $e^{-s t}$. Several applications of LT and DLT were discussed by many authors [6, 8-10]. The applications of the $n$-dimensional Laplace transform appear in heat equations, wave equations, and modeling in fluid dynamics $[13,14,21]$. In [22, 23], the authors considered some mathematical logistic models of fractional operators. Recently, the authors found the solutions of fractional difference equations [24-26]. Some more findings on fractional order models with the numerical simulation are discussed in [27-33]. For recent development in the theory of fractional difference operators, we refer to [15-20].

The LT and DLT are respectively defined as $\mathcal{L}[u(t)]=\int_{0}^{\infty} u(t) e^{-s t} d t$ and $\mathcal{L}[u(n)]=$ $\sum_{n=0}^{\infty} u(n) e^{-s n}, s>0$. From the basic difference identity $\left.\Delta^{-1} x_{n}\right|_{0} ^{\infty}=\sum_{n=0}^{\infty} x_{n}$ [4] the DLT can be expressed as $\mathcal{L}[u(n)]=\Delta^{-1} u(n) e^{-s n}$. In the literature the Laplace transform in discrete calculus comes from the time scale definition of the Laplace transform and has a strong relation with the $Z$-transform. Here we define a different kind of Laplace transform because it has two different kinds of solutions and is more suitable with the literature. Let $u(t)$ be an input signal (function), and let $h$ be a shift value. Then we define the alpha fractional

(c) The Author(s) 2020. This article is licensed under a Creative Commons Attribution 4.0 International License, which permits use, sharing, adaptation, distribution and reproduction in any medium or format, as long as you give appropriate credit to the original author(s) and the source, provide a link to the Creative Commons licence, and indicate if changes were made. The images or other third party material in this article are included in the article's Creative Commons licence, unless indicated otherwise in a credit line to the material. If material is not included in the article's Creative Commons licence and your intended use is not permitted by statutory regulation or exceeds the permitted use, you will need to obtain permission directly from the copyright holder. To view a copy of this licence, visit http://creativecommons.org/licenses/by/4.0/. 
frequency Laplace transform (LFT) with tuning factor $\alpha$ and frequency $s^{1 / v}$ as

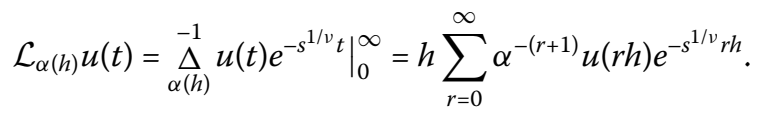

When $\alpha=1$ and $h=1$, transform (1) becomes the discrete Laplace transform. When $\alpha=1$ and $h \rightarrow 0$, (1) becomes the Laplace transform [5, 6]. To develop LFT, we can study the operators $\Delta_{h}$ and $\Delta_{\alpha(h)}$ and their inverses $[7,11]$.

In 2011, the authors in [1] defined the alpha difference operator as

$$
\underset{\alpha(h)}{\Delta} u(t)=\frac{u(t+h)-\alpha u(t)}{h}=u(t), \quad t \in[0, \infty), h \in(0, \infty) .
$$

In this research work, we extend the results on multiseries by using $\alpha$-difference operators and then analyze LFT for signals of algebraic and geometric type functions.

\section{Preliminaries}

In [3] the authors introduced $t_{h}^{(m)}=\prod_{r=0}^{m-1}(t-r h)$ and obtained the following expressions:
(i) $\quad \Delta_{h} t_{h}^{(m)}=(m h) t_{h}^{(m-1)}$,
(ii) $t_{h}^{(m)}=\sum_{r=1}^{m} s_{r}^{m} h^{m-r} t^{r}$,
(iii) $t^{m}=\sum_{r=1}^{m} S_{r}^{m} h^{m-r} t_{h}^{(r)}$,

where $s_{r}^{m}$ and $S_{r}^{m}$ denote the Stirling numbers.

Lemma 2.1 ([2]) If $\lim _{r_{i} \rightarrow \infty} \frac{1}{\alpha_{i}^{r_{i}}} \Delta_{\alpha_{i}\left(h_{i}\right)}^{-1} u\left(t+r_{i} h_{i}\right)=0$, then the $\alpha_{i}$-difference equation

$$
\underset{\alpha_{i}\left(h_{i}\right)}{\Delta} v(t)=u(t), \quad t \in[0, \infty), h_{i}>0,
$$

has a solution in the following infinite series form:

$$
\underset{\alpha_{i}\left(h_{i}\right)}{\stackrel{-1}{\Delta}} u(t)=\frac{-h_{i}}{\alpha_{i}} \sum_{r_{i}=0}^{\infty} \alpha_{i}^{-r_{i}} u\left(t+r_{i} h_{i}\right)
$$

Lemma 2.2 ([12]) Let $p>0$ and $1+\alpha^{2}-\cos p h \neq 0$. Then

$$
\underset{\alpha(h)}{\stackrel{-1}{\Delta}} \sin p t=h \frac{\sin p(t-h)-\alpha \sin p t}{1+\alpha^{2}-\cos p h}+c_{h(t)}
$$

and

$$
\underset{\alpha(h)}{\stackrel{-1}{\Delta}} \cos p t=h \frac{\cos p(t-h)-\alpha \cos p t}{1+\alpha^{2}-\cos p h}+c_{h(t)} . r .
$$




\section{Summation operator}

Remark 3.1 Hereafter we take $P=p\left(n_{1}-2 r_{1}\right)+q\left(n_{2}-2 r_{2}\right)$ and $\bar{P}=p\left(n_{1}-2 r_{1}\right)-q\left(n_{2}-2 r_{2}\right)$, so that $P$ and $\bar{P}$ depend on $n_{1}, n_{2}, r_{1}, r_{2}, p$, and $q$. We use the notation $\{(, o\}$ for odd numbers and $\{[, e\}$ for even numbers.

(i) $\mathbb{T}_{o o}=\{(1,0),(0,1)\}$,

(ii) $\mathbb{T}_{o e}=\{(1,0),(0,1),(1,1)\}$,

(iii) $\mathbb{T}_{e o}=\{(1,0),(0,1),(1,-1)\}$,

(iv) $\mathbb{T}_{e e}=\{(1,0),(0,1),(1,1),(1,-1)\}$,

(v) $\left(\left(n_{2}\right)\right)=\left(\begin{array}{c}n_{2} \\ \frac{n_{2}}{2}\end{array}\right)^{-u v\left(\frac{u-v}{u^{2}+v^{2}}\right)}$,

(vi) $\left(\left(n_{3}\right)\right)=\left(\begin{array}{c}n_{3} \\ \frac{n_{3}}{2}\end{array}\right) u v\left(\frac{u+v}{u^{2}+v^{2}}\right)$.

Here $s, p$ and $q$ are real numbers.

(1) For odd positive integers $n_{2}$ and $n_{3}$, we denote

$$
\sum_{\left(n_{2}, n_{3}\right)}^{s, c}=\frac{(-1)^{\frac{n_{2}-1}{2}}}{2^{n_{2}+n_{3}-1}} \sum_{s_{2}=0}^{\frac{n_{2}-1}{2}} \sum_{s_{3}=0}^{\frac{n_{3}-1}{2}}(-1)^{s_{2}} \frac{n_{2}^{\left(s_{2}\right)}}{s_{2} !} \frac{n_{3}^{\left(s_{3}\right)}}{s_{3} !} .
$$

(2) For even positive integers $n_{2}$ and $n_{3}$, we denote

$$
\sum_{\left[n_{2}, n_{3}\right]}^{s, c}=\frac{(-1)^{\frac{n_{2}}{2}}}{2^{n_{2}+n_{3}-1}} \sum_{s_{2}=0}^{\frac{n_{2}-2}{2}} \sum_{s_{3}=0}^{\frac{n_{3}-2}{2}}(-1)^{s_{2}} \frac{n_{2}^{\left(s_{2}\right)}}{s_{2} !} \frac{n_{3}^{\left(s_{3}\right)}}{s_{3} !} .
$$

The operator used for products of circular functions with exponential functions alone is defined as

$$
\sum_{\left(n_{2}, n_{3}\right)}^{s, c, m}=\frac{(-1)^{\frac{n_{2}-1}{2}}}{2^{n_{2}+n_{3}-1}} \sum_{s_{1}=0}^{m} \sum_{s_{2}=0}^{\frac{n_{2}-1}{2}} \sum_{s_{3}=0}^{\frac{n_{3}-1}{2}}(-1)^{s_{1}+s_{2}} \frac{m^{\left(s_{1}\right)}}{s_{1} !} \frac{n_{2}^{\left(s_{2}\right)}}{s_{2} !} \frac{n_{3}^{\left(s_{3}\right)}}{s_{3} !} .
$$

The operator used for products of circular functions with $\mathrm{t}$-factorial and also for products of circular, t-factorial, and exponential functions is defined as

$$
\sum_{n_{1}\left(n_{2}, n_{3}\right)}^{s, c, m+s_{1}}=\frac{(-1)^{\frac{n_{2}-1}{2}}}{2^{n_{2}+n_{3}-1}} \sum_{s_{1}=0}^{n_{1}} \sum_{s_{2}=0}^{\frac{n_{2}-1}{2}} \sum_{s_{3}=0}^{\frac{n_{3}-1}{2}} \sum_{s_{4}=0}^{m+s_{1}} \frac{n_{1}^{\left(s_{1}\right)} n_{2}^{\left(s_{2}\right)} n_{3}^{\left(s_{3}\right)}\left(m+s_{1}\right)^{\left(s_{4}\right)}}{(-1)^{s_{1}+s_{2}+s_{4}} s_{1} ! s_{2} ! s_{3} ! s_{4} !} .
$$

In the $m(\alpha)$-series formula, we use

$$
\sum_{(r)_{1} \rightarrow i}^{[t]}=\sum_{r_{1}=0}^{\left[\frac{t}{h}\right]} \sum_{r_{2}=0}^{\left[\frac{t-r_{1} h}{h}\right]} \ldots \sum_{r_{i}=0}^{\left[\frac{t-\left(r_{1}+r_{2} \ldots r_{i}\right) h}{h}\right]}, \quad(U V)=\left(\frac{u P+v \bar{P}}{u^{2}+v^{2}}\right) .
$$

\section{Multiseries inverse of product of two and three functions}

In this section, we derive a finite summation formula and $m$-series formula. Also, we present the $m$-series inverse of the product of two and three functions. 
Theorem 4.1 (Finite summation formula) Let $\alpha \neq 1$ and $m>1$. Then we have

$$
\sum_{r=1}^{m} \alpha^{r-1} h^{r} u(t-r h)={\underset{\alpha(h)}{\Delta}}_{\Delta}^{-1} u(t)-\alpha^{m+1} h^{m} \underset{\alpha(h)}{\stackrel{-1}{\Delta}} u(t-m h) .
$$

Note: (8) can be represented as $\left.\Delta_{\alpha(h)}^{-1} u(t)\right|_{t-m h} ^{t}$.

Proof From the definitions of $\Delta_{\alpha(h)}$ and $\Delta_{\alpha(h)}^{-1}$ we have

$$
\begin{aligned}
& \underset{\alpha(h)}{\Delta} v(t)=u(t) \text { implies } v(t)=\underset{\alpha(h)}{\Delta} u(t), \\
& \text { (i.e.) } \frac{v(t+h)-\alpha v(t)}{h}=u(t) \Rightarrow v(t+h)=h[u(t)+\alpha v(t)]
\end{aligned}
$$

Replacing $t$ by $t-h, t-2 h, \ldots, t-m h$ in (10), we get expressions for $v(t), v(t-h), \ldots, v(t-$ $m h)$. Successively substituting all these expressions into (10), we arrive at

$$
h u(t-h)+\alpha h^{2} u(t-2 h)+\cdots+\alpha^{m-1} h^{m} u(t-m h)=v(t)-\alpha^{m+1} h^{r} v(t-m h) .
$$

Now (8) follows from the equality $v(t)=\Delta_{\alpha(h)}^{-1} u(t-m h)$.

Remark 4.2 We denote $\Delta_{\alpha(h)}^{-1} u(t)-\alpha^{m+1} h^{r} \Delta_{\alpha(h)}^{-1} u(t-m h)=\left.\Delta_{\alpha(h)}^{-1} u(t)\right|_{t-m h} ^{t}$.

Lemma 4.3 Let $t \in[h, \infty), a^{h} \neq \alpha$, and $h(t)=(t-m h)$. Then we have

$$
\left.\underset{\alpha(h)}{\Delta} a^{t}\right|_{h(t)} ^{t}=\frac{h a^{t+h}}{\left(a^{h}-\alpha\right)}-\alpha^{m+1} h^{r+1} \frac{a^{h}}{\left(a^{h(t)}-\alpha\right)} .
$$

Proof Using (2) and Remark 4.2, we get (12).

Theorem 4.4 For the functions $u(t)$ and $v(t)$, we have

$$
\underset{\alpha(h)}{\Delta}[u(t) v(t)]=u(t) \underset{\alpha(h)}{\stackrel{-1}{\Delta}} v(t)-\underset{\alpha(h)}{\Delta}[\underset{\alpha(h)}{\Delta} v(t+h) \underset{h}{\Delta} u(t)] .
$$

Proof From the definition of $\Delta_{\alpha}(h)$ we have

$$
\underset{\alpha(h)}{\Delta}[u(t) w(t)]=u(t) \underset{\alpha(h)}{\Delta} w(t)+w(t+h) \underset{h}{\Delta} u(t)
$$

Now taking $\Delta_{\alpha(h)} w(t)=v(t)$ and $w(t)=\Delta_{\alpha(h)}^{-1} v(t)$ in equation (14), we obtain (13).

Theorem 4.5 (Product of two functions) For odd positive integers $n_{2}$ and $n_{3}$,

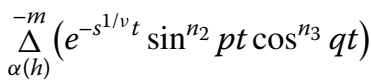

$$
\begin{aligned}
& =\sum_{\left(n_{2}, n_{3}\right)}^{s, c, m} \sum_{(u, v) \in \mathbb{T}_{o o}} \frac{e^{s_{1} 1^{1 / v} h}}{e^{s^{1 / v} t}} \frac{\alpha^{s_{1}} \sin (U V)\left(t-\left(m-s_{1}\right) h\right)}{\left(e^{-s^{1 / v} h}+\alpha^{2} e^{s^{1 / v} h}-2 \cos (U V) h\right)^{m}} .
\end{aligned}
$$


Proof After changing the powers of sin and cos into linear, we obtain

$$
\begin{aligned}
& \underset{\alpha(h)}{\Delta}\left(e^{-s^{1 / v} t} \sin ^{n_{2}} p t \cos ^{n_{3}} q t\right)=\sum_{\left(n_{2}, n_{3}\right)}^{s, c} \underset{\alpha(h)}{\Delta}\left(e^{-s^{1 / v} t}(\sin P t+\sin \bar{P} t)\right) \\
& =\operatorname{Im} \text { part of } \sum_{\left(n_{2}, n_{3}\right)}^{s, c} \times \underset{\alpha(h)}{\Delta}\left(e^{-s^{1 / v} t}\left(e^{i P t}+e^{i \bar{P} t}\right)\right) \\
& =\operatorname{Im} \text { part of } \sum_{\left(n_{2}, n_{3}\right)}^{s, c}\left(\frac{e^{\left(i P-s^{1 / v}\right) t}}{e^{\left(i P-s^{1 / v}\right) h}-\alpha}+\frac{e^{\left(i \bar{P}-s^{1 / v}\right) t}}{e^{\left(i \bar{P}-s^{1 / v}\right) h}-\alpha}\right) .
\end{aligned}
$$

After simplification, we get

$$
\begin{aligned}
& \stackrel{\Delta}{h}^{-1}\left(e^{-s^{1 / v} t} \sin ^{n_{2}} p t \cos ^{n_{3}} q t\right) \\
& \quad=\sum_{\left(n_{2}, n_{3}\right)}^{s, c, 1} \sum_{(u, v) \in \mathbb{T}_{o o}} e^{-s^{1 / v} t} \alpha^{s_{1}} e^{s_{1} s^{1 / v} h} \frac{\sin (U V)\left(t-\left(1-s_{1}\right) h\right)}{\left(e^{-s^{1 / v} h}+\alpha^{2} e^{s^{1 / v} h}-2 \cos (U V) h\right)^{m}} .
\end{aligned}
$$

Applying $\Delta_{\alpha(h)}^{-1}$ to both sides of equation (16), we get

$$
\begin{aligned}
\underset{\alpha(h)}{\Delta}\left(e^{-s^{1 / v} t} \sin ^{n_{2}} p t \cos ^{n_{3}} q t\right) \\
\quad=\sum_{\left(n_{2}, n_{3}\right)}^{s, c, 2} \sum_{(u, v) \in \mathbb{T}_{o o}} e^{-s^{1 / v} t} \alpha^{s_{1}} e^{s_{1} s^{1 / v} h} \frac{\sin (U V)\left(t-\left(2-s_{1}\right) h\right)}{\left(e^{-s^{1 / v} h}+\alpha^{2} e^{s^{1 / v} h}-2 \cos (U V) h\right)^{m}} .
\end{aligned}
$$

Continuing this process up to $m$-inverse, we get (15).

Theorem 4.6 (Product of three functions) For odd positive integers $n_{2}$ and $n_{3}$,

$$
\begin{aligned}
& \underset{\alpha(h)}{\Delta}\left(t_{h}^{\left(n_{1}\right)} e^{-s^{1 / v} t} \sin ^{n_{2}} p t \cos ^{n_{3}} q t\right) \\
& \quad=\sum_{n_{1}\left(n_{2}, n_{3}\right)}^{s, c, m+s_{1}} \sum_{(u, v) \in \mathbb{T}_{o o}} \frac{t_{h}^{\left(n_{1}-s_{1}\right)} m_{\left(s_{1}\right)} \alpha^{s_{4}}}{h^{-s_{1}} e^{s^{1 / v}\left(t+s_{1} h\right)}} \frac{e^{s_{4} s^{1 / v} h} \sin (U V)\left(t-\left(m-s_{4}\right) h\right)}{\left(e^{-s^{1 / v} h}+\alpha^{2} e^{s^{1 / v} h}-2 \alpha \cos (U V) h\right)^{m+s_{1}}} .
\end{aligned}
$$

Proof Let $f_{1}(t)=t_{h}^{(1)} e^{-s^{1 / v} t} \sin ^{n_{2}} p t \cos ^{n_{3}} q t$

$$
\begin{aligned}
\underset{\alpha(h)}{\Delta} f_{1}(t) & =\sum_{\left(n_{2}, n_{3}\right)}^{s, c} \underset{\alpha(h)}{\Delta}\left(t_{h}^{(1)} e^{-s^{1 / v} t}(\sin P t+\sin \bar{P} t)\right) \\
& =\sum_{1\left(n_{2}, n_{3}\right)}^{s, c, 1+s_{1}} \sum_{(u, v) \in \mathbb{T}_{o o}} \frac{t_{h}^{\left(1-s_{1}\right)} 1_{\left(s_{1}\right)} \alpha^{s_{4}}}{h^{-s_{1}} e^{s^{1 / v}\left(t+s_{1} h\right)}} \frac{e^{s_{4} s^{1 / v} h} \sin (U V)\left(t-\left(1-s_{4}\right) h\right)}{\left(e^{-s^{1 / v} h}+\alpha^{2} e^{s^{1 / v} h}-2 \alpha \cos (U V) h\right)^{1+s_{1}}} .
\end{aligned}
$$

Applying $\Delta_{h}^{-1}$ to both sides, we get

$$
\underset{h}{-2} f_{1}(t)=\sum_{1\left(n_{2}, n_{3}\right)}^{s, c, 2+s_{1}} \sum_{(u, v) \in \mathbb{T}_{o o}} \frac{t_{h}^{\left(1-s_{1}\right)} 2_{\left(s_{1}\right)} \alpha^{s_{4}}}{h^{-s_{1}} e^{s^{1 / v}\left(t+s_{1} h\right)}} \frac{e^{s_{4} s^{1 / v} h} \sin (U V)\left(t-\left(2-s_{4}\right) h\right)}{\left(e^{-s^{1 / v} h}+\alpha^{2} e^{s^{1 / v} h}-2 \alpha \cos (U V) h\right)^{2+s_{1}}}
$$


Continuing this process, we get

$$
\underset{\alpha(h)}{\Delta} f_{1}(t)=\sum_{1\left(n_{2}, n_{3}\right)}^{s, c, m+s_{1}} \sum_{(u, v) \in \mathbb{T}_{o o}} \frac{t_{h}^{\left(1-s_{1}\right)} m_{\left(s_{1}\right)} \alpha^{s_{4}}}{h^{-s_{1}} e^{s^{1 / v}\left(t+s_{1} h\right)}} \frac{e^{s_{4} s^{1 / v} h} \sin (U V)\left(t-\left(m-s_{4}\right) h\right)}{\left(e^{-s^{1 / v} h}+\alpha^{2} e^{s^{1 / v} h}-2 \alpha \cos (U V) h\right)^{m+s_{1}}} .
$$

Similarly, we can obtain

$$
\begin{aligned}
& \underset{\alpha(h)}{\Delta}\left(t_{h}^{(2)} e^{-s^{1 / v} t} \sin ^{n_{2}} p t \cos ^{n_{3}} q t\right) \\
& \quad=\sum_{2\left(n_{2}, n_{3}\right)}^{s, c, m+s_{1}} \sum_{(u, v) \in \mathbb{T}_{o o}} \frac{t_{h}^{\left(2-s_{1}\right)} m_{\left(s_{1}\right)} \alpha^{s_{4}}}{h^{-s_{1}} e^{s^{1 / v}\left(t+s_{1} h\right)}} \frac{e^{s_{4} s^{1 / v} h} \sin (U V)\left(t-\left(m-s_{4}\right) h\right)}{\left(e^{-s^{1 / v} h}+\alpha^{2} e^{s^{1 / v} h}-2 \alpha \cos (U V) h\right)^{m+s_{1}}} .
\end{aligned}
$$

Continuing this process up to $m$-inverse for $n_{1}$, we get equation (17).

Corollary 4.7 For odd positive integers $n_{2}$ and $n_{3}$, we have

$$
\begin{aligned}
\underset{\alpha(h)}{\Delta}\left(t^{n_{1}} e^{-s^{1 / v} t} \sin ^{n_{2}} p t \cos ^{n_{3}} q t\right) \\
=\sum_{r_{1}=1}^{n_{1}} \sum_{n_{1}\left(n_{2}, n_{3}\right)}^{s, c, m+s_{1}} \sum_{(u, v) \in \mathbb{T}_{o o}} \frac{S_{r_{1}}^{n_{1}} t_{h}^{\left(r_{1}-s_{1}\right)} m_{\left(s_{1}\right)} \alpha^{s_{4}}}{h^{r_{1}-n_{1}} h^{-s_{1}} e^{s^{1 / v}\left(t+s_{1} h\right)}} \\
\quad \times \frac{e^{s_{4} s^{1 / v} h} \sin (U V)\left(t-\left(m-s_{4}\right) h\right)}{\left(e^{-s^{1 / v} h}+\alpha^{2} e^{s^{1 / v} h}-2 \alpha \cos (U V) h\right)^{m+s_{1}}} .
\end{aligned}
$$

Proof The proof follows by applying (ii) of (3) in Theorem 4.6.

Corollary 4.8 For odd positive integers $n_{2}$ and $n_{3}$, we have

$$
\begin{aligned}
& \underset{\alpha(h)}{\stackrel{-m}{\Delta}}\left(t_{h}^{\left(n_{1}\right)} \sin ^{n_{2}} p t \cos ^{n_{3}} q t\right) \\
& \quad=\sum_{n_{1}\left(n_{2}, n_{3}\right)}^{s, c, m+s_{1}} \sum_{(u, v) \in \mathbb{T}_{o o}} t_{h}^{\left(n_{1}-s_{1}\right)} m_{\left(s_{1}\right)} h^{s_{1}} \alpha^{s_{4}} \frac{\sin (U V)\left(t-\left(m-s_{4}\right) h\right)}{\left(1+\alpha^{2}-2 \alpha \cos (U V) h\right)^{m+s_{1}}} .
\end{aligned}
$$

Theorem 4.9 ( $m$-series formula) For $m \in \mathbb{N}(2)$ and $t \in[m h, \infty)$, we have

$$
\begin{aligned}
& \sum_{r=0}^{\left[\frac{t}{h}\right]} \frac{(r+1)_{m-1}}{(m-1) !} \alpha^{r} u(t-r h)+\sum_{i=1}^{m-1} \sum_{(r)_{1 \rightarrow i}}^{[t]} \alpha^{\left[\frac{t-\sum_{j=1}^{i} r_{j} h}{h}\right]+\sum_{j=1}^{i} r_{j}+1} \\
& \quad \times{\underset{\alpha(h)}{\Delta} u(h(t)+(m-i-1) h)}^{-(m-i)} \\
& =\underset{\alpha(h)}{\Delta} u(t+m h)-\alpha^{\left[\frac{t}{h}\right]+1} \underset{\alpha(h)}{\Delta} u(h(t)+(m-1) h) .
\end{aligned}
$$

Proof Replacing $t$ by $t-h$ in (8) and multiplying both sides by $\alpha$, we get

$$
\begin{aligned}
& \alpha\left\{u(t-h)+\alpha u(t-2 h)+\alpha^{2} u(t-3 h)+\cdots+\alpha^{\left[\frac{t-h}{h}\right]} u(h(t))\right\} \\
& \quad=\alpha\left\{\underset{\alpha(h)}{\Delta} u(t)-\alpha^{\left[\frac{t-h}{h}\right]+1} \underset{\alpha(h)}{\Delta} u(h(t))\right\} .
\end{aligned}
$$


Replacing $t$ by $t-2 h$ in (8) and multiplying both sides by $\alpha^{2}$, we get

$$
\begin{gathered}
\alpha^{2}\left\{u(t-2 h)+\alpha u(t-2 h)+\alpha^{2} u(t-3 h)+\cdots+\alpha^{\left[\frac{t-2 h}{h}\right]} u(h(t))\right\} \\
=\alpha^{2}\left\{\underset{\alpha(h)}{\Delta} u(t-h)-\alpha^{\left[\frac{t-2 h}{h}\right]+1} \underset{\alpha(h)}{\Delta} u(h(t))\right\} .
\end{gathered}
$$

Proceeding similarly, replacing $t$ by $h(t)$ in (8), and multiplying both sides by $\alpha^{\left[\frac{t}{h}\right]}$, we get $\alpha^{\left[\frac{t}{h}\right]} u(h(t))=\alpha^{\left[\frac{t}{h}\right]}\left\{\Delta_{\alpha(h)}^{-1} u(h(t)+h)-\alpha^{\left[\frac{h(t)}{h}\right]+1} \Delta_{\alpha(h)}^{-1} u(h(t))\right\}$.

Adding all left- and right-hand sides with (8), we get

$$
\begin{aligned}
& \sum_{r=0}^{\left[\frac{t}{h}\right]}(r+1) \alpha^{r} u(t-r h)+\sum_{r_{1}=0}^{\left[\frac{t}{h}\right]} \alpha^{\left[\frac{t-r_{1} h}{h}\right]+r_{1}+1} \underset{\alpha(h)}{\Delta} u(h(t)) \\
& \quad=\underset{\alpha(h)}{\Delta} u(t+2 h)-\alpha^{\left[\frac{t}{h}\right]+1} \underset{\alpha(h)}{\Delta} u(h(t)+h) .
\end{aligned}
$$

Similarly, by replacing $t$ by $t-h, t-2 h, \ldots, h(t)$ in (21), multiplying by $\alpha, \alpha^{2}, \ldots \alpha^{\left[\frac{t}{h}\right]}$, respectively, and adding all with (21), we arrive at

$$
\begin{aligned}
& \sum_{r=0}^{\left[\frac{t}{h}\right]} \frac{(r+1)(r+2)}{2 !} \alpha^{r} u(t-r h)+\sum_{r_{1}=0}^{\left[\frac{t}{h}\right]} \alpha^{\left[\frac{t-r_{1} h}{h}\right]+r_{1}+1} \underset{\alpha(h)}{\Delta} u(h(t)+h)+\sum_{r_{1}=0}^{-2} \sum_{r_{2}=0}^{\left[\frac{t}{h}\right]}\left[\frac{t-r_{1} h}{h}\right] \\
& \quad \times \alpha^{\left[\frac{t-\left(r_{1}+r_{2}\right) h}{h}\right]+r_{1}+r_{2}+1} \underset{\alpha(h)}{\Delta} u(h(t)) \\
& =\underset{\alpha(h)}{\Delta} u(t+3 h)-\alpha^{\left[\frac{t}{h}\right]+1} \underset{\alpha(h)}{\Delta} u(h(t)+2 h) .
\end{aligned}
$$

Proceeding similarly, we finally obtain (20).

Theorem 4.10 For odd positive integers $n_{1}$ and $n_{2}$, the $m$-series corresponding to (15) is

$$
\begin{aligned}
& \sum_{r=0}^{\left[\frac{t}{h}\right]} \frac{(r+1)_{(m-1)}}{(m-1) !} \alpha^{r}(t-r h)_{h}^{\left(n_{1}\right)} e^{-s^{1 / v}(t-r h)} \sin ^{n_{2}} p(t-r h) \cos ^{n_{3}} q(t-r h) \\
& +\sum_{i=0}^{m-1} \sum_{(r)_{1 \rightarrow i}} \sum_{n_{1}\left(n_{2}, n_{3}\right)}^{s, c, m-i+s_{1}} \sum_{(u, v) \in \mathbb{T}_{o o}} \alpha^{\left[\frac{t-\sum_{j=1}^{i} r_{j} h}{h}\right]+\sum_{j=1}^{i} r_{j} h+1}\left(h\left(t_{1}\right)\right)_{h}^{\left(n_{1}-s_{1}\right)} \alpha^{s_{4}} \\
& \times \frac{(m-i)_{\left(s_{1}\right)} h^{s_{1}}}{e^{s^{1 / v}\left(h\left(t_{1}\right)+s_{1} h\right)}} \frac{e^{s_{4} s^{1 / v} h} \sin (U V)\left(h(t)+\left(s_{4}-i-1\right) h\right)}{\left(e^{-s^{1 / v} h}+\alpha^{2} e^{s^{1 / v} h}-2 \alpha \cos (U V) h\right)^{m-i+s_{1}}} \\
& =\sum_{n_{1}\left(n_{2}, n_{3}\right)}^{s, c, m+s_{1}} \sum_{(u, v) \in \mathbb{T}_{o o}}\left\{\frac{(t+m h)_{h}^{\left(n_{1}-s_{1}\right)} m_{\left(s_{1}\right)}}{h^{-s_{1}} e^{s^{1 / v}\left((t+m h)+s_{1} h\right)}} \frac{\alpha^{s_{4}} e^{s_{4} s^{1 / v} h} \sin (U V)\left(t+s_{4} h\right)}{\left(e^{-s^{1 / v} h}+\alpha^{2} e^{s^{1 / v} h}-2 \alpha \cos (U V) h\right)^{m+s_{1}}}\right. \\
& \left.-\alpha^{\left[\frac{t}{h}\right]+1} \frac{\left(h\left(t_{2}\right)\right)_{h}^{\left(n_{1}-s_{1}\right)} m_{\left(s_{1}\right)}}{h^{-s_{1}} e^{s^{1 / v}\left(h\left(t_{2}\right)+s_{1} h\right)}} \frac{\alpha^{s_{4}} e^{s_{4} s^{1 / v} h} \sin (U V)\left(h(t)+\left(s_{4}-1\right) h\right)}{\left(e^{-s^{1 / v} h}+\alpha^{2} e^{s h}-2 \alpha \cos (U V) h\right)^{m+s_{1}}}\right\},
\end{aligned}
$$

where $h\left(t_{1}\right)=h(t)+(m-(i+1) h), h\left(t_{2}\right)=h(t)+(m-1) h$. 
Proof The proof is obtained by replacing $u(t)$ by $\left(t_{h}^{\left(n_{1}\right)} e^{-s^{1 / v} t} \sin ^{n_{2}} p t \cos ^{n_{3}} q t\right)$ in Theorem 4.9 and using (20).

Example 4.11 Consider (20), where $m=3, p=5, q=3, \alpha=2, s=2, n_{1}=4, n_{2}=4, n_{3}=4$, $P=\left(5\left(4-2 r_{1}\right)+3\left(4-2 r_{2}\right)\right)$, and $\bar{P}=\left(5\left(4-2 r_{1}\right)-3\left(4-2 r_{2}\right)\right)$,

$$
\begin{aligned}
& \text { LHS }=\sum_{r=0}^{\left[\frac{t}{h}\right]} \frac{(r+1)_{(3-1)}}{(3-1) !} \alpha^{r}(t-r h)_{h}^{(4)} e^{-s^{1 / v}(t-r h)} \sin ^{4} 5(t-r h) \cos ^{4} 3(t-r h) \\
& +\sum_{i=1}^{m-1} \sum_{(r)_{1 \rightarrow i}}^{[t]} \alpha^{\left[\frac{t-\sum_{j=1}^{i} r_{j} h}{h}\right]+\sum_{j=1}^{i} r_{j}+1} \underset{\alpha(h)}{\Delta} u(h(t)+(m-i-1) h) \\
& =\underset{\alpha(h)}{\Delta} u(t+m h)-\alpha^{\left[\frac{t}{h}\right]+1} \underset{\alpha(h)}{\Delta} u(h(t)+(m-1) h) .
\end{aligned}
$$

Here

$$
\begin{aligned}
u(t)= & \sum_{4\left[n_{2}, n_{3}\right)}^{s, c, 3+s_{1}} \sum_{(u, v) \in \mathbb{T}_{e e}} \frac{\left(\left(n_{2}\right)\right)\left(\left(n_{3}\right)\right) t_{h}^{\left(4-s_{1}\right)}}{h^{-s_{1}} e^{s^{1 / v}\left(t+s_{1} h\right)}} \frac{\alpha^{s_{4}} e^{s_{4} s^{1 / v} h} h \cos (U V)\left(t-\left(3-s_{4}\right) h\right)}{\left(e^{-s^{1 / v} h}+\alpha^{2} e^{s^{1 / v} h}-2 \alpha \cos (U V) h\right)^{3+s_{1}}} \\
& +\frac{n_{2}^{\left(\frac{n_{2}}{2}\right)}}{\frac{n_{2}}{2} !} \frac{n_{3}^{\left(\frac{n_{3}}{2}\right)}}{\frac{n_{3}}{2} !} \frac{1}{2\left(e^{-s^{1 / v} h}-\alpha\right)^{3+s_{1}}} .
\end{aligned}
$$

\section{Laplace transforms and its applications}

Here,] we derive the fractional frequency Laplace transform for the input functions (signals) in multiseries $(m=1)$ and analyze the results by MATLAB.

Theorem 5.1 For odd positive integers $n_{2}$ and $n_{3}$,

(i)

$$
\begin{aligned}
L_{\alpha(h)}\left(\sin ^{n_{2}} p t \cos ^{n_{3}} q t\right) & =-h \sum_{r=0}^{\infty}\left(\alpha^{-(r+1)} e^{-s^{1 / v} r h} \sin ^{n_{2}} p r h \cos ^{n_{3}} q r h\right) \\
& =\sum_{\left(n_{2}, n_{3}\right)}^{s, c, 1} \sum_{(u, v) \in \mathbb{T}_{o o}} \frac{-h e^{s_{1} s^{1 / v}} \alpha^{s_{1}} \sin (U V)\left(s_{1}-1\right) h}{\left(e^{-s^{1 / v} h}+\alpha^{2} e^{s^{1 / v} h}-2 \cos (U V) h\right)},
\end{aligned}
$$

(ii)

$$
\begin{aligned}
& { }_{t} L_{\alpha(h)}\left(\sin ^{n_{2}} p t \cos ^{n_{3}} q t\right) \\
& =-h \times \sum_{r=0}^{\infty}\left(\alpha^{-(r+1)} e^{-s^{1 / v}(t+r h)} \sin ^{n_{2}} p(t+r h) \cos ^{n_{3}} q(t+r h)\right) \\
& =h \sum_{\left(n_{2}, n_{3}\right)}^{s, c, 1} \sum_{(u, v) \in \mathbb{T}_{o o}} \frac{e^{s_{1} s^{1 / v} h}}{e^{s^{1 / v}} t} \frac{\alpha^{s_{1}} \sin (U V)\left(t-\left(1-s_{1}\right) h\right)}{\left(e^{-s^{1 / v} h}+\alpha^{2} e^{s^{1 / v} h}-2 \cos (U V) h\right)} .
\end{aligned}
$$

Proof Taking the limit from 0 to $\infty$ in (15) gives the Laplace transform of $\sin ^{n_{2}} p t \cos ^{n_{3}} q t$.

Similarly, we can find results for other cases (odd-even, even-odd, even-even).

In the following example, we analyze LTT using MATLAB. 


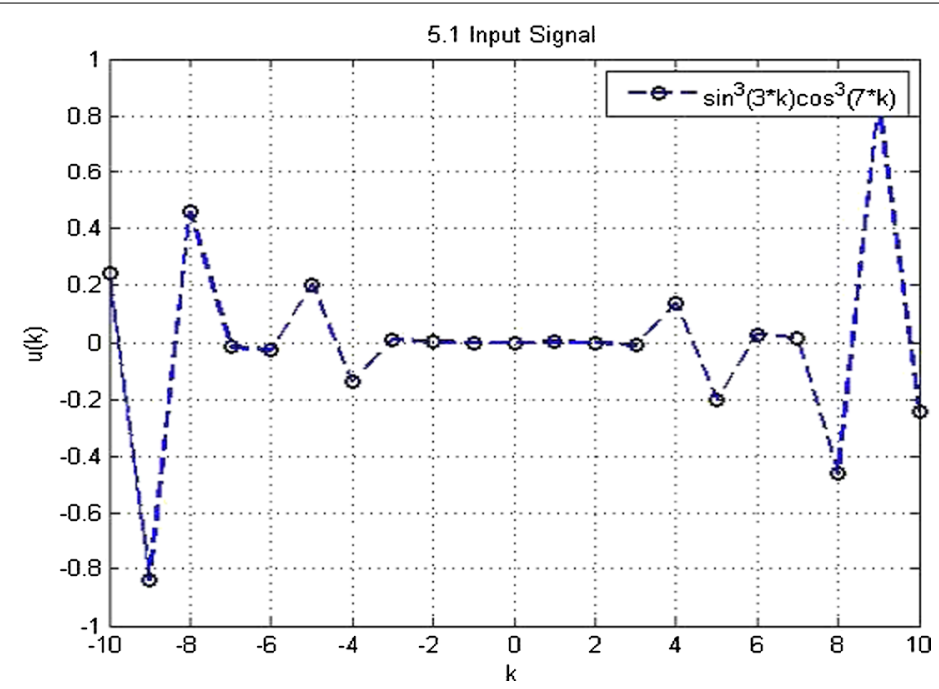

Figure 1 Input Signal

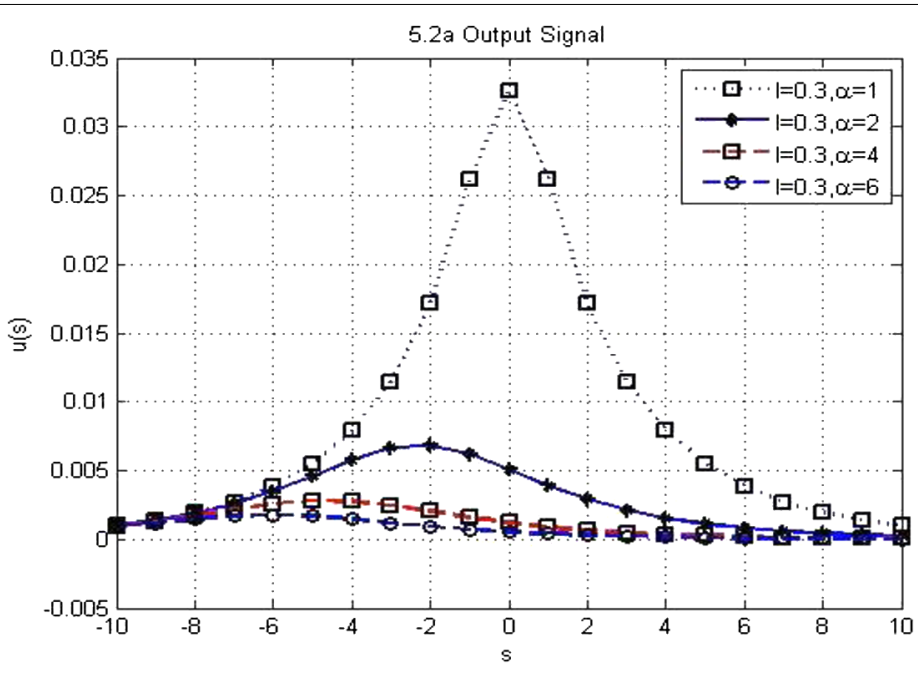

Figure 2 Output signal for $\ell=0.3$ and $\alpha$

Example 5.2 Taking $n_{2}=3, n_{3}=3, p=3$, and $q=7$ in Theorem 5.1, we obtain

$$
\begin{aligned}
L_{\alpha(h)}\left(\sin ^{3} 3 t \cos ^{3} 7 t\right) & =(-h) \sum_{r=0}^{\infty} \alpha^{-(r+1)} e^{-s^{1 / v} r h} \sin ^{3} 3 r h \cos ^{3} 7 r h \\
& =\sum_{(3,3)}^{s, c, 1} \sum_{(u, v) \in \mathbb{T}_{o o}} \frac{-h e^{s_{1} s^{1 / n u}} h \alpha^{s_{1}} \sin (U V)\left(s_{1}-1\right) h}{\left(e^{-s^{1 / v} h}+\alpha^{2} e^{s^{1 / v} h}-2 \cos (U V) h\right)},
\end{aligned}
$$

which is verified for $v=0.1, \alpha=4, h=0.5$, and $s=10$ by MATLAB.

The results are analyzed with input and output signals. Figure 1 shows the input signal (function) for the product of sine and cosine functions. Figure 2 shows the output signal 


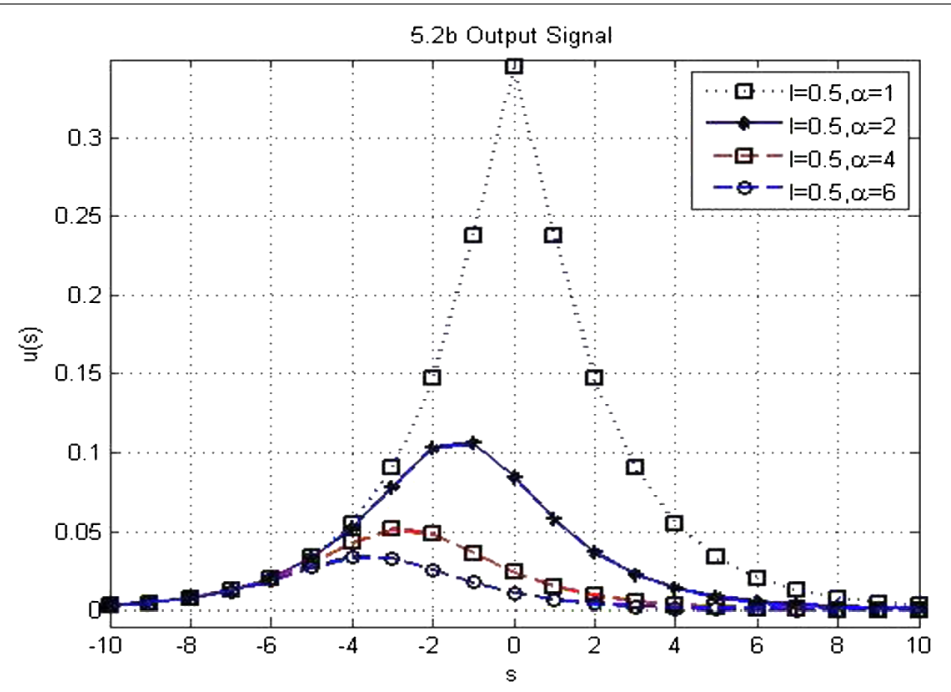

Figure 3 Output signal for $\ell=0.5$ and $\alpha$

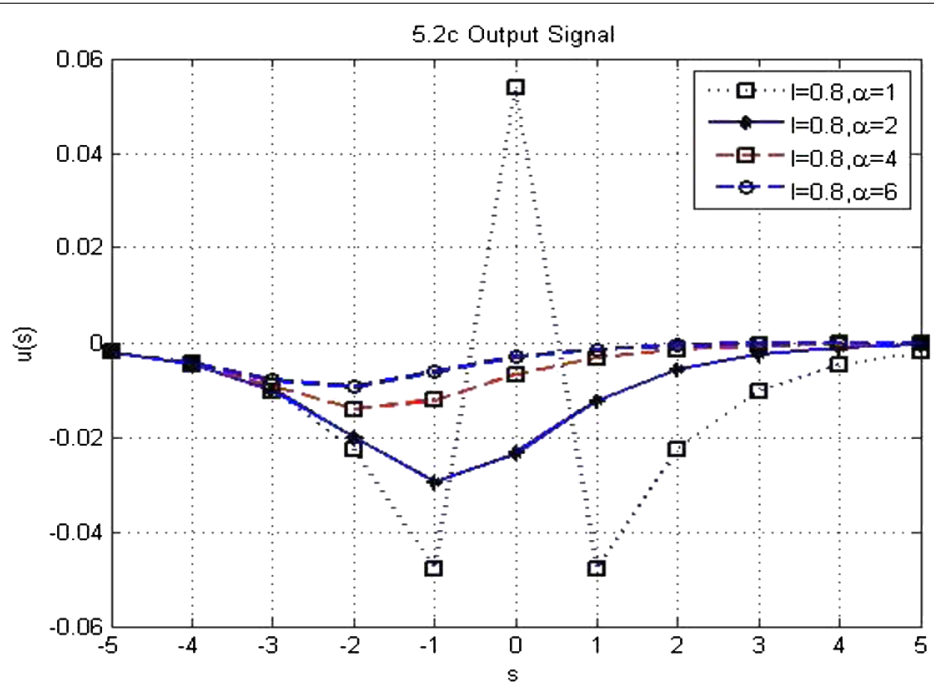

Figure 4 Output signal for $\ell=0.8$ and $\alpha$

for $\ell=0.3$ and varying $\alpha$. Figure 3 shows the output signal for $\ell=0.5$ with varying $\alpha$. Figure 4 is the output signal for $\ell=0.8$ with varying $\alpha$.

Theorem 5.3 For odd positive integers $n_{2}$ and $n_{3}$, we have

(i)

$$
\begin{aligned}
L_{\alpha(h)} & \left(t^{n_{1}} \sin ^{n_{2}} p t \cos ^{n_{3}} q t\right) \\
= & -h \sum_{r=0}^{\infty}\left(\alpha^{-(r+1)}(r h)^{n_{1}} e^{-s^{1 / v} r h} \sin ^{n_{2}} p r h \cos ^{n_{3}} q r h\right) \\
= & \sum_{r_{1}=1}^{n_{1}} \sum_{n_{1}\left(n_{2}, n_{3}\right)}^{s, c, 1+r_{1}} \sum_{(u, v) \in \mathbb{T}_{o o}} \frac{S_{r_{1}}^{n_{1}} h^{n_{1}-r_{1}} 1_{\left(r_{1}\right)} \alpha^{s_{4}}}{h^{-r_{1}} e^{1 / v} r_{1} h}
\end{aligned}
$$




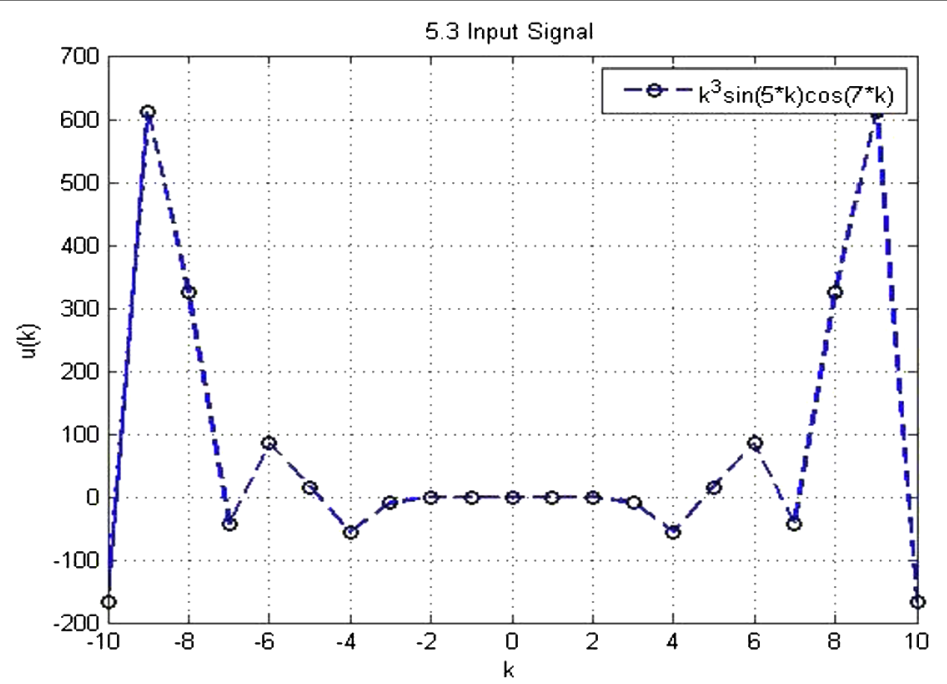

Figure 5 Input signal

$$
\times \frac{e^{s_{4} s^{1 / v} h} \sin (U V)\left(s_{4}-1\right) h}{\left(e^{-s^{1 / v} h}+\alpha^{2} e^{s^{1 / v} h}-2 \alpha \cos (U V) h\right)^{1+r_{1}}}
$$

(ii)

$$
\begin{aligned}
{ }_{t} L_{\alpha(h)} & \left(t^{n_{1}} \sin ^{n_{2}} p t \cos ^{n_{3}} q t\right) \\
= & -h \times \sum_{r=0}^{\infty}\left(\alpha^{-(r+1)}(t+r h)^{n_{1}} e^{-s^{1 / v}(t+r h)} \sin ^{n_{2}} p(t+r h) \cos ^{n_{3}} q(t+r h)\right) \\
= & \sum_{r_{1}=1}^{n_{1}} \sum_{n_{1}\left(n_{2}, n_{3}\right)}^{s, c, 1+s_{1}} \sum_{(u, v) \in \mathbb{T}_{o o}} \\
& \times \frac{S_{r_{1}}^{n_{1}} t_{h}^{\left(r_{1}-s_{1}\right)} 1_{\left(s_{1}\right)} \alpha^{s_{4}}}{h^{r_{1}-n_{1}} h^{-s_{1}} e^{s^{1 / v}\left(t+s_{1} h\right)}} \frac{e^{s_{4} s^{1 / v} h} \sin (U V)\left(t-\left(1-s_{4}\right) h\right)}{\left(e^{-s^{1 / v} h}+\alpha^{2} e^{s^{1 / v} h}-2 \alpha \cos (U V) h\right)^{1+s_{1}}} .
\end{aligned}
$$

Proof Taking the limit 0 to $\infty$ in (18) gives the Laplace transform of $t^{n_{1}} \sin ^{n_{2}} p t \cos ^{n_{3}} q t$

Similarly, we can find results for other cases (odd-even, even-odd, even-even).

Example 5.4 Taking $n_{1}=3, n_{2}=1, n_{3}=1, p=5$, and $q=7$ in Theorem 5.3, we obtain

$$
\begin{aligned}
& L_{\alpha(h)}\left(t^{3} \sin 5 t \cos 7 t\right) \\
& =(-h) \sum_{r=0}^{\infty} \alpha^{-(r+1)}(r h)^{3} e^{-s^{1 / v} r h} \sin 5 r h \cos 7 r h \\
& =\sum_{r_{1}=1}^{3} \sum_{3(1,1)}^{s, c, 1+r_{1}} \sum_{(u, v) \in \mathbb{T}_{o o}} \frac{S_{r_{1}}^{3} h^{3-r_{1}} 1_{\left(r_{1}\right)} \alpha^{s_{4}}}{h^{-r_{1}} e^{s^{1 / v} r_{1} h}} \frac{e^{s_{4} s^{1 / v} h} \sin (U V)\left(s_{4}-1\right) h}{\left(e^{-s^{1 / v} h}+\alpha^{2} e^{s^{1 / v} h}-2 \alpha \cos (U V) h\right)^{1+r_{1}}},
\end{aligned}
$$

which is verified for $\alpha=5, h=0.8, v=0.1$, and $s=15$ by MATLAB.

The results are analyzed with input and output signals. Figure 5 shows the input signal (function) for the product of polynomial, sine, and cosine functions. Figure 6 


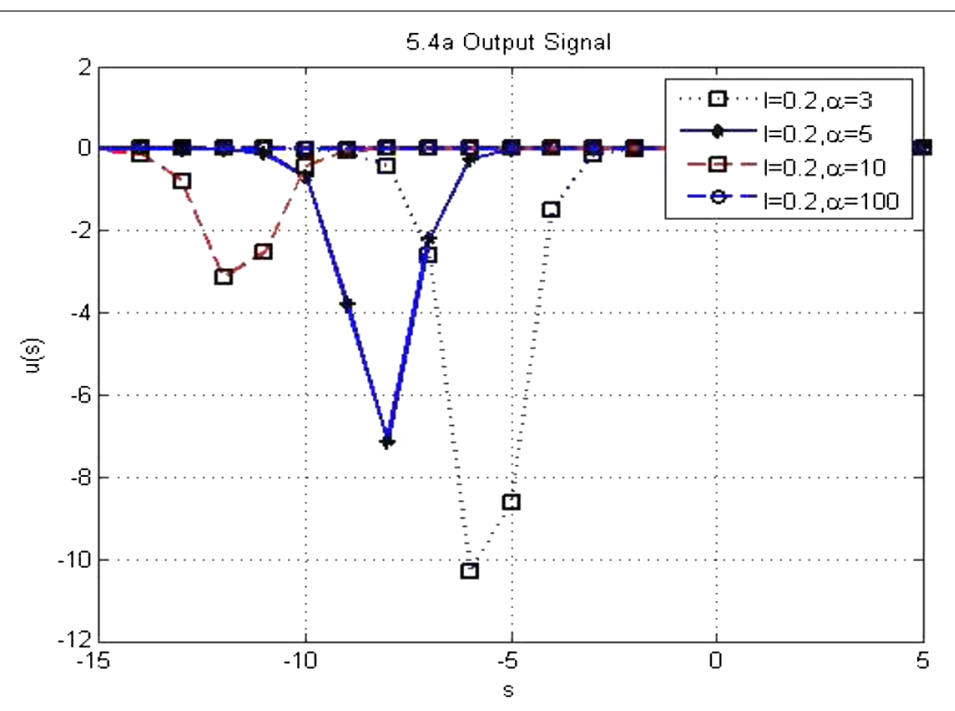

Figure 6 Output signal for $\ell=0.2$ and $\alpha$

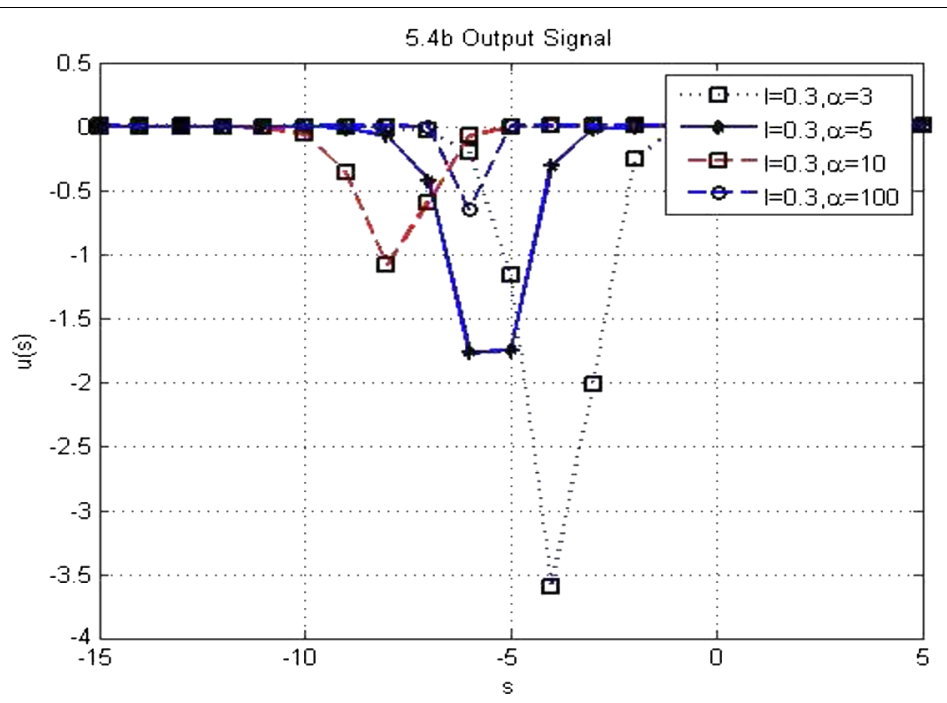

Figure 7 Output signal for $\ell=0.3$ and $\alpha$

shows the output signal for $\ell=0.2$ with varying $\alpha$. Figure 7 shows the output signal for $\ell=0.3$ and varying $\alpha$. Figure 8 shows the output signal for $\ell=0.8$ with varying $\alpha$.

\section{Conclusions}

We proposed formulas for the frequency Laplace transforms of the products of two and three functions and a multiseries formula for circular functions. Further, LFT is employed on circular functions to get appropriate results numerically and also analyzed the findings for different values of tuning factor $\alpha$ and fractional frequency factor $s^{1 / v}$. We also observed with the help of the diagrams generated by MATLAB that LFT gives innumerable outcomes for the given input signal, and this enables us to make a choice for an optimal 


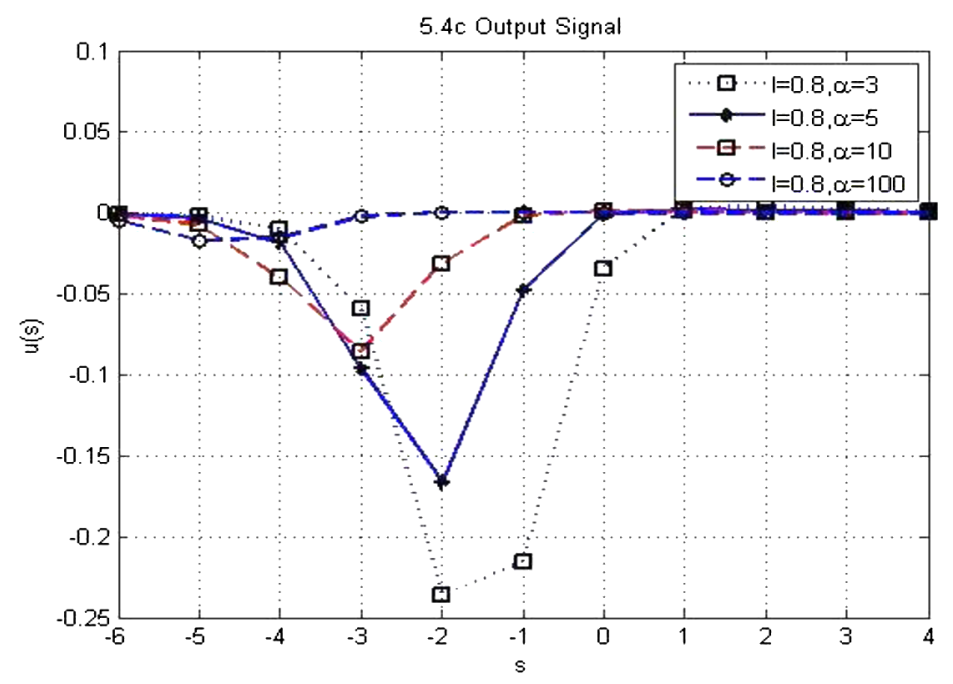

Figure 8 Output signal for $\ell=0.8$ and $\alpha$

one. As a very important findingof this research, when $\alpha=v=1$, we get the Laplace transform existing in the literature.

\section{Acknowledgements}

The second author would like to thank Prince Sultan University for funding this work through research group Nonlinear Analysis Methods in Applied Mathematics (NAMAM), group number RG-DES-2017-01-17.

\section{Funding}

Catalyzed and financially supported by Tamilnadu State Council for Science and Technology, Dept. of Higher Education, Government of Tamilnadu.

\section{Availability of data and materials}

Please contact author for data requests.

\section{Competing interests}

All the authors declare that they have no competing interests.

\section{Authors' contributions}

All authors contributed equally and significantly in writing this paper. All authors read and approved the final manuscript.

\section{Author details}

'Department of Mathematics, Sacred Heart College (Autonomous), Tirupattur, India. ${ }^{2}$ Department of Mathematics and General Sciences, Prince Sultan University, Riyadh, Saudi Arabia. ${ }^{3}$ Department of Medical Research, China Medical University, Taichung, Taiwan. ${ }^{4}$ Department of Computer Science and Information Engineering, Asia University, Taichung,

Taiwan. ${ }^{5}$ Department of Mathematics, Çankaya University, Ankara, Turkey.

\section{Publisher's Note}

Springer Nature remains neutral with regard to jurisdictional claims in published maps and institutional affiliations.

Received: 9 February 2020 Accepted: 12 May 2020 Published online: 24 May 2020

\section{References}

1. Maria Susai Manuel, M., Chandrasekar, V., Britto Antony Xavier, G.: Solutions and applications of certain class of $\alpha$-difference equations. Int. J. Appl. Math. 24(6), 943-954 (2011)

2. Maria Susai Manuel, M., Dominic Babu, G., Britto Antony Xavier, G.: Finite and infinite multi-series type solutions of generalized mixed difference equation. Int. J. Pure Appl. Math.

3. Britto Antony Xavier, G., Sathya, S., Vasanthakumar, S.U.: m-series of the generalized difference equation to circular functions. Int. J. Math. Arch. 4(7), 200-209 (2013)

4. Agarwal, R.P.: Difference Equations and Inequalities. Marcel Dekker, New York (2000)

5. Akansu, A.N., Poluri, R.: Walsh-like nonlinear phase orthogonal codes for direct sequences CDMA communications. IEEE Trans. Signal Process. 55, 3800-3806 (2007)

6. Ivic, A.: Some applications of Laplace transforms in analytic number theory. Novi Sad J. Math. 45(1), 31-44 (2015) 
7. Britanak, V., Rao, K.R.: The fast generalized discrete Fourier transforms: a unified approach to the discrete sinusoidal transforms computation. Signal Process. 79, 135-150 (1999)

8. Ivic, A.: The Laplace transform of the fourth moment of of the zeta-function. Publ. Elektroteh. Fak. Univ. Beogr., Mat. $11,41-48(2000)$

9. Sedletskii, A.M.: Fourier Transforms and Approximations. Gordon \& Breach, Amsterdam (2000)

10. Jutila, M.: The Mellin transform of the square of Riemann's zeta-function. Period. Math. Hung. 42, 179-190 (2001)

11. Maria Susai Manuel, M., Britto Antony Xavier, G., Chandrasekar, V., Pugalarasu, R.: Theory and applicaton of the generalized difference operator of the $n$th kind (part I). Demonstr. Math. XLV(1), 95-106 (2012)

12. Britto, A., Xavier, G., Nasira Begum, H.: $m(\alpha)$-series to circular functions using power set notation. International Organization Of Scientific Research 10. Issue: 5, Ver. IV (Sep-Oct. 2014), PP 34-43

13. Buschman, R.G.: Heat transfer between a fluid and a plate: multidimensional Laplace transformation methods. Int. J. Math. Math. Sci. 6(3), 589-596 (1983)

14. Dahiya, R.S., Vinayagamoorthy, M.: Laplace transform pairs of $n$-dimensions and heat conduction problem. Math. Comput. Model. 13(10), 35-50 (1990)

15. Abdeljawad, T.: On delta and nabla Caputo fractional differences and dual identities. Discrete Dyn. Nat. Soc. 2013, Article ID 406910 (2013)

16. Abdeljawad, T.: Dual identities in fractional difference calculus within Riemann. Adv. Differ. Equ. 2013, 36 (2013)

17. Abdeljawad, T., Jarad, F., Baleanu, D.: A semigroup-like property for discrete Mittag-Leffler functions. Adv. Differ. Equ.

18. Abdeljawad, T:: Different type kernel $h$-fractional differences and their fractional $h$-sums. Chaos Solitons Fractals 116 , $146-156$ (2018)

19. Suwan, I., Owies, S., Abdeljawad, T.: Monotonicity results for $h$-discrete fractional operators and application. Adv. Differ. Equ. 2018, 207 (2018)

20. Abdeljawad, T., Al-Mdallal, Q.M.: Discrete Mittag-Leffler kernel type fractional difference initial value problems and Gronwall's inequality. J. Comput. Appl. Math. 339, 218-230 (2018) Special Issue. https://doi.org/10.1016/j.cam.2017.10.021

21. Saberi-Nadjafi, J.: Theorems on N-dimensional inverse Laplace transformations. In: Proc. of the Eighth Annual Conference on Applied Mathematics, Oklahoma, pp. 317-330 (1992)

22. Abdeljawad, T., Hajji, M.A., Al-Mdallal, Q.M., Jarad, F.: Analysis of some generalized ABC—fractional logistic models. Alex. Eng. J. (2020). https://doi.org/10.1016/j.aej.2020.01.030

23. Abdeljawad, T., Al-Mdallal, Q.M., Jarad, F.: Fractional logistic models in the frame of fractional operators generated by conformable derivatives. Chaos Solitons Fractals 119, 94-101 (2019)

24. Haq, F., Shah, K., Al-Mdallal, Q.M., Jarad, F.: Application of a hybrid method for systems of fractional order partial differential equations arising in the model of the one-dimensional Keller-Segel equation. Eur. Phys. J. Plus 134(9), 461 (2019)

25. Abu-Saris, R., Al-Mdallal, Q.: On the asymptotic stability of linear system of fractional-order difference equations. Fract. Calc. Appl. Anal. 16(3), 613-629 (2013)

26. Abdeljawad, T., Al-Mdallal, Q.M., Hajji, M.A.: Arbitrary order fractional difference operators with discrete exponential kernels and applications. Discrete Dyn. Nat. Soc. (2017). https://doi.org/10.1155/2017/4149320

27. Khan, A., Gomez-Aguilar, J.F., Abdeljawad, T., Khan, A.: Dynamical study of fractional order mutualism parasitism food web module. Chaos Solitons Fractals 134, 109685 (2020)

28. Khan, A., Gomez-Aguilar, J.F., Abdeljawad, T., Khan, A.: Stability and numerical simulation of a fractional order plant nectar pollinator model. Alex. Eng. J. 59, 49-59 (2020)

29. Khan, H., Tunc, C., Khan, A.: Green function's properties and existence theorems for nonlinear singular delay fractional differential equations. Discrete Contin. Dyn. Syst., Ser. S (2020). https://doi.org/10.3934/dcdss.2020139

30. Khan, H., Gomez-Aguilar, J.F., Alkhazzan, A., Khan, A.: A fractional order HIV-TB coinfection model with nonsingular Mittag-Leffler law. Math. Methods Appl. Sci. 43, 3786-3806 (2020)

31. Khan, H., Khan, A., Jarad, F., Shah, A.: Existence and data dependence theorems for solutions of an ABC-fractional order impulsive system. Chaos Solitons Fractals 131, 109477 (2019)

32. Khan, A., Khan, A., Gomez-Aguilar, J.F., Abdeljawad, T.: Existence and Hyers-Ulam stability for a nonlinear singular fractional differential equations with Mittag-Leffler kernel. Chaos Solitons Fractals 127, 422-427 (2019)

33. Khan, H., Jarad, F., Abdeljawad, T., Khan, A.: A singular ABC-fractional differential equation with $p$-Laplacian operator. Chaos Solitons Fractals 129, 56-61 (2019)

\section{Submit your manuscript to a SpringerOpen ${ }^{\circ}$ journal and benefit from:}

- Convenient online submission

- Rigorous peer review

- Open access: articles freely available online

- High visibility within the field

Retaining the copyright to your article

Submit your next manuscript at $\gg$ springeropen.com 